NASZA DERMATOLOGIA Online OUR DERMATOLOGY Online

Source of Support:

Georgia Dermatopathology

Associates, Atlanta, Georgia, USA

(MSH, AMAV).

Competing Interests:

None

\section{IMMUNOHISTOCHEMISTRY STUDIES IN A CASE OF DERMATITIS HERPETIFORMIS DEMONSTRATE COMPLEX PATTERNS OF REACTIVITY}

\author{
Ana Maria Abreu Velez ${ }^{1,3}$, Jorge Oliver $^{2}$, Michael S. Howard ${ }^{1}$ \\ ${ }^{1}$ Georgia Dermatopathology Associates, Atlanta, Georgia, USA \\ ${ }^{2}$ Hamilton Medical Center Pathology, Dalton, Georgia, USA \\ ${ }^{2}$ Section of Dermatology, University of Antioquia, Medellin, Colombia, South America
}

Corresponding author: Ana Maria Abreu Velez, MD PhD

abreuvelez@yahoo.com

\begin{abstract}
Introduction: Dermatitis herpetiformis (DH) is an autoimmune, clinically pleomorphic, papulovesicular disorder sometimes associated with celiac disease and gluten sensitivity. DH is categorized by subepidermal vesicles and bullae on hematoxylin and eosin (H\&E) staining, and with immunoglobulin A deposits present along the dermal papillary tips on direct immunofluorescence (DIF).

Case Report: We describe a 50 year old female that presented with sudden onset pruritus and clinical blisters, predominantly on extensor areas of the extremities. Biopsies for H\&E examination, as well as immunohistochemistry (IHC) and DIF analysis were performed.

Results: H\&E examination demonstrated subepidermal blistering; within the blister lumen, numerous neutrophils were present, with occasional eosinophils and lymphocytes also seen. DIF examination revealed linear deposits of IgA along the epidermal basement membrane zone, associated with other immunoglobulins and complement. IHC examination showed similar patterns of reactivity to IgA, and also to other immunoreactants. Cells positive for CDla were present within the blisters, correlating with S-100 staining. Cells staining positive for CD8, CD45 and occasionally CD4 and Granzyme B were seen not only in the blister lumens, but also around neurovascular packages under the blisters. Finally, CD2 positive cells were found around the upper dermal blood vessels.

Discussion: Focal DIF linear IgA deposition is the classic hallmark diagnostic finding in DH. However, it is possible that genetic susceptibility and environmental triggers also play a crucial role in the pathogenesis, often acting via cellular pathways exhibiting disease-associated polymorphisms. In tolerance breakthrough, the initiating antigen presenting cells likely lead to immune system cell differentiation, and activation of adaptive immunity.
\end{abstract}

Key words: dermatitis herpetiformis; immunohistochemistry; autoimmunity

Abbreviations and acronyms: Dermatitis herpetiformis (DH), immunohistochemistry (IHC), direct and indirect immunofluorescence (DIF and IIF), hematoxylin and eosin (H\&E), basement membrane zone (BMZ).

Cite this article:

Ana Maria Abreu Velez, Jorge Oliver, Michael S. Howard. Howard: Immunohistochemistry studies in a case of dermatitis herpetiformis demonstrate complex patterns of reactivity. Our Dermatol Online. 2013; 4(Suppl.3): 627-630.

\section{Introduction}

Dermatitis herpetiformis (DH) was initially described by Louis A. Duhring, M.D. (1845-1913) [1]. DH is a rare disease characterized by inflammatory pustules, usually grouped on extensor surfaces. Previously, groups of researchers have reported autoantibodies deposited along the dermal papillary tip basement membrane zones (BMZ). Other groups have reported autoantibodies to tissue transglutaminase and epidermal transglutaminase, as well as anti-endomysial antibodies [2-5]. In some clinical cases, a gluten-free diet may ameliorate the disease. The treatments of choice are dapsone and sulfones $[6,7]$.
Clinical, histopathologic and direct immunofluorescence (DIF) studies have been used in the diagnosis of this disease. DIF studies have demonstrated that the main autoreactivity in $\mathrm{DH}$ is found along the dermal papillary tip BMZ, with IgA present in a focal linear pattern of "snow on the mountain tops", this concept has prevailed over time [2,3]. We aim to report multiple other positive immunoreactants in our case in addition to $\operatorname{IgA}$.

\section{Case Report}

A 50 year old female presented with sudden onset of pruritus and clinical blisters, initially located on the extremities. 
These lesions then extended to the rest of the body, associated with diffuse patches of erythema containing microvesiculation. The lesions initially prevailed on extensor areas of the extremities, although some vesicles were seen on the back. Biopsies for hematoxylin and eosin (H\&E), immunohistochemistry (IHC) and direct immunofluorescence (DIF) analysis were performed as previously described [8-12].

\section{Result}

DIF

In brief, skin cryosections were prepared, and incubated with multiple fluorescein isothiocyanate (FITC) conjugated antibodies as previously reported [8-12].

\section{IHC}

Paraffin-embedded sections (3-4 micron thickness) were used for routine $\mathrm{H} \& \mathrm{E}$ staining and for immunohistochemistry. IHC was performed utilizing a Dako (Carpinteria, California, U.S.A.) EnVision detection system and the immunoperoxidase method. The following Dako primary monoclonal antibodies were utilized: CD1a, CD2, CD4, CD8, CD45, IgG, IgM, IgD, IgE, Complement/C1q, Complement/C3, Complement/C4, myeloid/histiocyte antigen, S-100, Granzyme B, kappa light chains, lambda light chains, fibrinogen and albumin. We also utilized Factor XIIIa antibodies, obtained from Biocare Medical, Concord, California, U.S.A. Our IHC studies were performed as previously described [8-12].

\section{Microscopic Description}

Examination of the H\&E tissue sections demonstrated a subepidermal blistering disorder. Specifically, the epidermis displayed mild, diffuse spongiosis; subepidermal blistering was present, with numerous neutrophils noted within the blister lumens. Occasional luminal eosinophils were also present. The papillary dermis contained a mild, superficial, perivascular infiltrate of lymphocytes, histiocytes and neutrophils; eosinophils were rare. We also observed the presence of multiple erythrocytes displaying a "rouleaux process" in dermal blood vessels subjacent to the blisters (Fig. 1). No epidermal dyskeratosis or acantholysis was noted.

DIF demonstrated focal, linear IgA deposition on the dermal papillary tips. However, we also found deposits of $\operatorname{IgG}, \operatorname{IgM}$, IgD, Kappa light chains, Lambda light chains, Complement/ $\mathrm{C} 1 \mathrm{q}$, Complement/C3 and fibrinogen in a diffuse pattern; specifically, these immunoreactants were noted in linear deposits along the BMZ, and within the upper dermis (Fig. 1). By IHC, the patterns of positivity were also not restricted to the tips of the dermal papillae, but also present in the upper dermis (Fig. 1, 2). In addition to this reactivity, we also were able to see a clear neurovascular reactivity around dermal eccrine sweat ducts with the same immunoreactants. We observed diffuse positivity with CD8 and CD45 in these areas; focal CD4 and Granzyme B positivity were also appreciated (Fig. 1, 2). CD2 positive cells were found around most of the upper dermal blood vessels subjacent to the blisters, with some positive cells present in the blister lumens.

CD1a and S100 stains were also positive above the blisters in the epidermis (Fig. 1); the myeloid/histiocyte antigen staining was exclusively located in papillary dermal areas (Fig. 1). Finally, Factor XIIIa positive staining was accentuated around most of the upper dermal blood vessels.
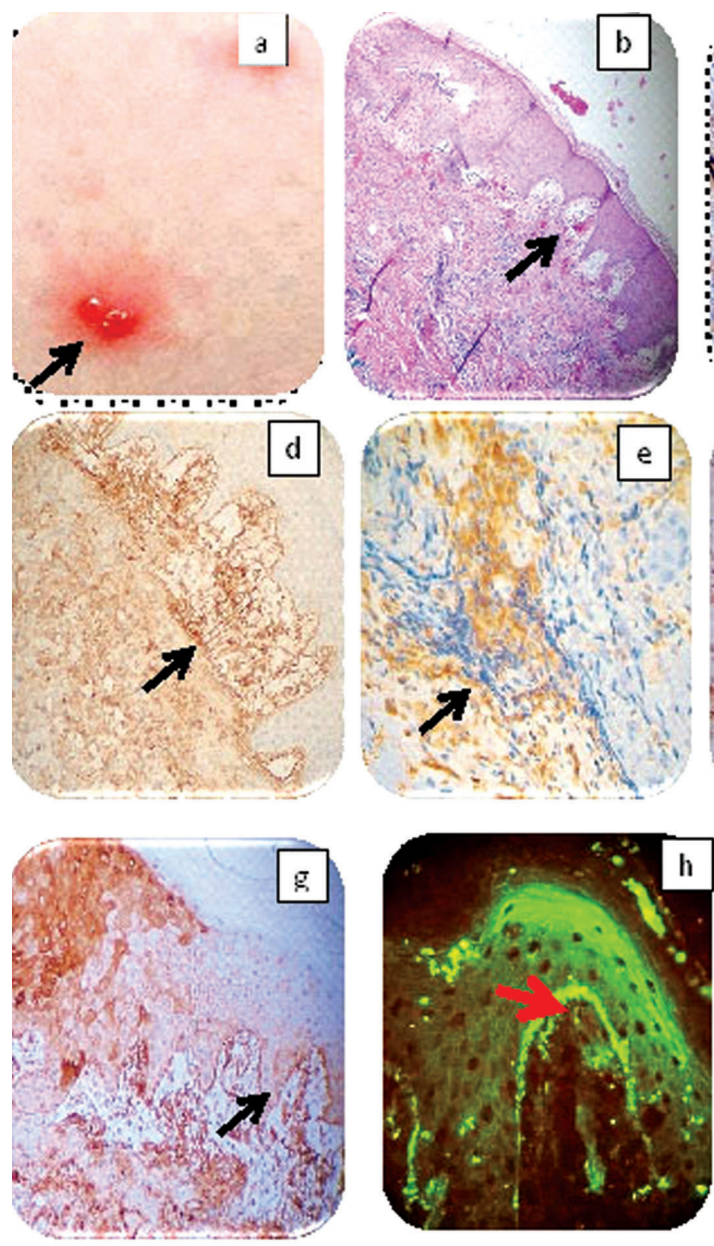

Figure 1. a. A representative clinical lesion of a DH patient; small blisters on an erythematous base (black arrow), note some are grouped. b. Representative H\&E section, showing a subepidermal blister with a luminal inflammatory infiltrate (black arrow) (100x). c. IHC, demonstrating IgG epidermal keratinocytic pericytoplasmic, intracytoplasmic and nuclear staining (brown staining; black arrow). d. Same as c, but in this case highlighting IgG staining at the BMZ (brown staining; black arrow) and additional staining in the blister lumen (200x). e. IHC positive IgG staining of the upper dermal neurovascular plexus (brown staining; black arrow). f. IHC positive $\operatorname{IgE}$ staining against the same neurovascular plexus as in e(brown staining; black arrow). g. IHC, highlighting positive IgD staining in several keratinocytes above a subepidermal blister, and within the blister (brown staining; black arrow). h. DIF, demonstrating positive staining with FITC conjugated $\operatorname{IgA}$ antibody (green staining; red arrow) (200x). i. IHC, demonstrating positive staining for myeloid/ histiocyte antigen around a dermal papillary tip blood vessel (brown staining; black arrow). 

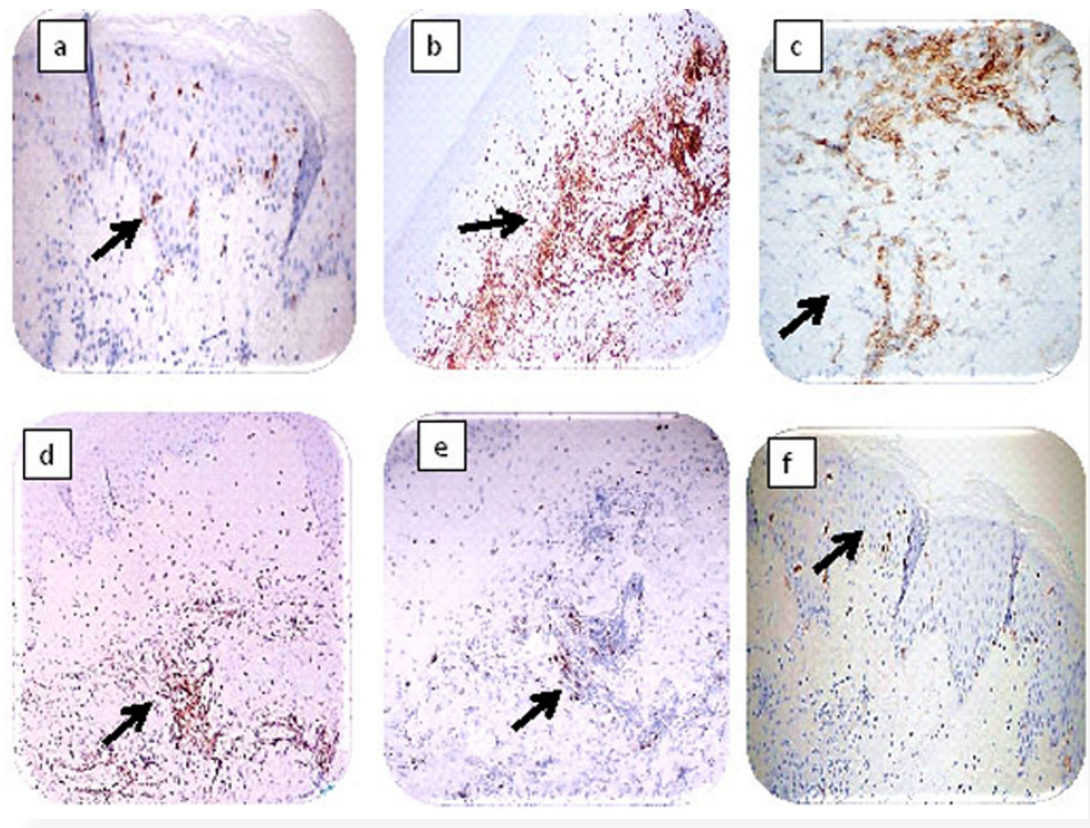

Figure 2. a. Positive IHC staining for CD1a, present in the epidermis above a subepidermal blister (black arrows, brown stain). b. IHC CD45 positive staining, accentuated around upper and lower dermal blood vessels (brown staining; black arrow)(100x). c. CD4 positive IHC staining in a dermal neurovascular area (brown staining; black arrow). d. CD8 positive IHC staining in a region spanning the upper and lower dermal neurovascular packages (brown staining; black arrow). e. Positive IHC staining for Granzyme B, present in focal cells around a dermal neurovascular package (brown staining; black arrow). f. Positive S-100 IHC staining on cells within the epidermis above a subepidermal blisters (brown staining; black arrow).

\section{Discussion}

Currently, the diagnosis of DH is based on clinical and histopathologic data, as well as DIF findings of focal, linear deposits of IgA in the dermal papillary tip BMZs. In our case we noted these traditional DIF findings, as well as evidence of other immunoglobulins and complement components as immunoreactants. In addition, we were able to document several types of antigen presenting cells present around the blistering areas. The additional immune response cells included $\mathrm{T}$ lymphocytes. IgA class switching has been previously reported to occur via both $\mathrm{T}$ lymphocyte dependent and $\mathrm{T}$ lymphocyte independent pathways, further, the IgA antibody response targets both pathogenic and commensal microorganisms. As described in the literature, we found the H\&E histologic presence of a neutrophilic infiltrate in blister lumens near the basement membrane zone (BMZ). We further noted the presence of dermal blood vessel erythrocytes in rouleaux formation; we suggest that increased viscosity could be present in thin blood vessels of the upper dermis. Finally, the significance in our case of abundant CD2, CD8 and CD45 staining (and in a few areas, some CD4 and Granzyme B positive cells) around the upper dermal blood dermal vessels and selected neurovascular packages remains unknown.

In $\mathrm{DH}$, the documented loss of tolerance seen in spontaneous human autoimmunity was traditionally believed to be restricted to autoantibody responses produced by B lymphocytes. We have previously reported other cases where T lymphocytes and antigen presenting cells are abundant in lesional skin from DH patients [13]. IHC analysis has been demonstrated to be of value in studying DH cases [14]. Other groups have also described a complex autoimmunity in $\mathrm{DH}$; this autoimmunity may vary among patients, especially in the presence of celiac disease, lymphoproliferative disorders, other systemic autoimmune disorders and/or endomysial antibodies [15].

Based on our current knowledge of immunology and autoimmune responses, several mechanisms may be at play in $\mathrm{DH}$; possibilities include 1) loss of tolerance by $\mathrm{T}$ cells,
2) T lymphocyte/B lymphocyte discordance, 3) aberrant $B$ lymphocyte receptor mediated feedback, 4) molecular mimicry, 5) idiotypic cross reactions, 6) epitope modification and 7) cryptic epitope exposure. In one endemic form of pemphigus foliaceus, we have previously documented a complex immune response involving multiple immunoglobulins and complement components [16].

We suggest that in DH, a substitution of the heavy chain constant regions of $\operatorname{IgM}$ and $\operatorname{IgD}$ with that of $\operatorname{IgG}, \operatorname{Ig} \mathrm{A}$ or $\operatorname{IgE}$ could occur. Such immunoglobulin class switching would endow antibodies with novel effectors functions, thus enhancing the ability of the immune system to effectively perform a complex immune response. The presence of such class switching is suggested by studies on other diseases [17]. More DH cases are needed for further studies, in order to address these etiologic possibilities.

\section{Acknowledgement}

Jonathan S. Jones, HT(ASCP), at Georgia Dermatopathology Associates for excellent technical assistance.

\section{REFERENCES}

1. Duhring LA: Dermatitis herpetiformis. JAMA. 1983;250:212-6.

2. Zone JJ, Meyer LJ, Petersen MJ: Deposition of granular IgA relative to clinical lesions in dermatitis herpetiformis. Arch Dermatol. 1996;132:912-8.

3. Dieterich W, Laag E, Bruckner-Tuderman L, Reunala T, Kárpáti $\mathrm{S}$, Zágoni T, et al: Antibodies to tissue transglutaminase as serologic markers in patients with dermatitis herpetiformis. J Invest Dermatol. 1999;113:133-6.

4. Preisz K, Sárdy M, Horváth A, Kárpáti S: Immunoglobulin, complement and epidermal transglutaminase deposition in the cutaneous vessels in dermatitis herpetiformis. J Eur Acad Dermatol Venereol. 2005;19:74-9.

5. Sárdy M, Kárpáti S, Merkl B, Paulsson M, Smyth N: Epidermal transglutaminase (TGase 3) is the autoantigen of dermatitis herpetiformis. J Exp Med. 2002;195:747-7. 
6. Frödin T, Gotthard R, Hed J, Molin L, Norrby K, Walan A: Gluten-free diet for dermatitis herpetiformis: the long-term effect on cutaneous, immunological and jejunal manifestations. Acta Derm Venereol. 1981;61:405-411.

7. Mendes FB, Hissa-Elian A, Abreu MA, Gonçalves VS: Review: dermatitis herpetiformis. An Bras Dermatol. 2013;88:594-9.

8. Abreu-Velez AM, Howard MS, Yi H, Gao W, Hashimoto T: Grossniklaus HE. Neural system antigens are recognized by autoantibodies from patients affected by a new variant of endemic pemphigus foliaceus in Colombia. J Clin Immunol. 2011;31:356-68. 9. Abreu Velez AM, Yi H, Googe PB Jr, Mihm MC Jr, Howard MS: Autoantibodies to melanocytes and characterization of melanophages in patients affected by a new variant of endemic pemphigus foliaceus. J Cutan Pathol. 2011;38:710-19.

10. Abreu Velez AM, Howard MS, Brzezinski P: Immunofluorescence in multiple tissues utilizing serum from a patient affected by systemic lupus erythematosus. Our Dermatol Online. 2012;3:36-42.

11. Abreu Velez AM, Howard MS, Hashimoto K, Hashimoto T: Autoantibodies to sweat glands detected by different methods in serum and in tissue from patients affected by a new variant of endemic pemphigus foliaceus. Arch Dermatol Res. 2009;301:711-8.

12. Abreu-Velez AM, Yepes-Naranjo MM, Avila IC, Londoño ML, Googe PB, Velásquez-Velez JE, et al: Tissue inhibitor of metalloproteinase 1, matrix metalloproteinase 9, alpha-1 antitrypsin, metallothionein and urokinase type plasminogen activator receptor in skin biopsies from patients affected by autoimmune blistering diseases. Our Dermatol Online. 2013;4:275-80.
13. Abreu Velez AM, Yi H, Girard JG, Zhe J, Duque-Ramírez M, Arias LF, et al: Dermatitis herpetiformis bodies and autoantibodies to noncutaneous organs and mitochondria in dermatitis herpetiformis. Our Dermatol Online. 2012;3:283-91.

14. Pfaltz K, Mertz K, Rose C, Scheidegger P, Pfaltz M, Kempf W: C3d immunohistochemistry on formalin-fixed tissue is a valuable tool in the diagnosis of bullous pemphigoid of the skin. J Cutan Pathol. 2010;37:654-8.

15. Alonso-Llamazares J, Gibson LE, Rogers RS III: Clinical, pathologic, and immunopathologic features of dermatitis herpetiformis: review of the Mayo Clinic experience. Int J Dermatol. 2007;46:910-9.

16. Selmi C: Autoimmunity in 2012. Clin Rev Allergy Immunol. 2013;45:290-301.

17. Cerutti A, Qiao X, He B: Plasmacytoid dendritic cells and the regulation of immunoglobulin heavy chain class switching. Immunol Cell Biol. 2005;83:554-62. 